\title{
The current status on the UV upturn
}

\author{
Sukyoung K. Yi ${ }^{1}$ \\ ${ }^{1}$ Yonsei University, Department of Astronomy, Seoul 120-749, Republic of Korea \\ email: yi@yonsei.ac.kr
}

\begin{abstract}
The UV upturn phenomenon found in giant elliptical galaxies through space observations has been a mystery. Recent GALEX observations have revealed new facts. The most notable is the rarity of UV upturn galaxies. Unlike previous beliefs, UV upturn is found only in less than 10 percent of giant ellitpical galaxies. Another notable finding is that the UV flux has been increasing for the last couple of billion years. This is consistent with the theoretical predictions that suggest hot horizontal branch stars are the main UV sources. Remaining theoretical and observational issues on this topic can be found in another recent review of mine (Yi 2008).
\end{abstract}

Keywords. galaxies: elliptical and lenticular, $\mathrm{cD}$, galaxies: evolution

\section{Prologue}

I recently reviewed this topic (Yi 2008) and recommend readers to refer to it for a more complete and complementary review. More historic reviews can be found elsewhere (e.g., Greggio \& Renzini 1999; O'Connell 1999). In this partial review, I would like to stress on the two recent observational discoveries, both made by the Galaxy Evolution Explorer $(G A L E X)$.

\section{UV upturn is rare!}

Previous UV studies on elliptical galaxies were based on the pointing observations for a limited number of neaby galaxies. It seemed that most of the giant (roughly speaking, super- $L *$ ) elliptical galaxies exhibit a UV flux that is unaccountably high for their high metallicity and age. Theorists were challenged by this ubiquity of the UV upturn phenomenon and were forced to find an answer that works for all such galaxies. Since giant ellipticals are generally believed to be predominantly-old stelar populations, evolved hot horizontal-branch stars became the most obvious candidate culprits (e.g., Greggio \& Renzini 1990; Horch et al. 1988; Bressan, Chiosi, \& Fagotto 1994; Dorman, O'Connell, \& Rood 1995; Yi, Demarque, \& Kim 1997).

On the other hand, a growingly large number of observational studies are revealing the complex star formation histories of elliptical galaxies (e.g., Trager et al. 2000). At least 15 percent of nearby giant ellipticals are believed to have had recent star formation (RSF) and this fraction seems to rise dramatically as galaxy mass decreases (Yi 2005; Kaviraj et al. 2007; Schawinski et al. 2007). This causes trouble to the theorists who have been trying to find hot star solutions in dominantly old populations.

All this opaque has now been cleared out by the GALEX survey (Martin et al. 2005). Unlike previous investigations, GALEX detected tens of thousands of nearby $(z<0.2)$ ellipticals. According to the GALEX observations, only 10 percent (or fewer) of the nearby giant elliptical galaxies show UV spectral shapes that are consistent with the general definition of the UV upturn. The majority of giant ellipticals are UV weak, and greater than 15 percent show a sign of recent star formation. UV upturn is no longer a ubiquitous but a rare phenomenon in old populations. Interestingly, small ellipticals 
do not show a clear UV upturn. Theories must now explain why only a small fraction of ellipticals show a UV upturn. An interesting scenario based on helium sedimentation preferentially occuring in brightest cluster galaxies has recently been suggested (Peng \& Nagai 2009). While this scenario is not without problems, it poses an interesting possibility. The old challenge of the presumed ubiquity is now gone, but a newer and tougher challenge has appeared.

\section{UV upturn evolves!}

The current favourite, hot horizontal-branch theories for the UV upturn predict that only very old stellar populations develop a pronounced UV upturn. Hence, the strength of the UV upturn has been suggested to be a strong function of time (Yi et al. 1999). Alternatively, models based on the binary origin predicted no substantial evolution in the UV flux ever since shortly after the galaxy formation (Han et al. 2007). The early data on a galaxy cluster at $z=0.375$ from HST/FOC appeared to suggest no evolution (Brown et al. 1998). However, the data used in that study was later declared to be dubious by the original authors.

We finally have observational data to test these predictions. Lee et al. (2005) and Ree et al. (2007) obtained the FUV data of the brightest elliptical galaxies in rich galaxy clusters reaching upto $z \sim 0.2$ and found that the UV strength has indeed been rising for the last couple of billion years. In their works, they show how extremely difficult it is to obtain good signal-to-noise data for passively-evolving (hence UV-quiet) galaxies upto redshift 0.2 and how important it is to distinguish the UV flux from old stars from the UV flux from young ones. Only with an extra-careful analysis making use of multiband images and spectroscopic data can one remove contaminants of galaxies with young stars or nuclear activities. When only the robustly-passive galaxies are used, there appears to be a clear trend of rising UV flux with age.

The recent work of Atlee et al. (2009) attempts to achieve a reliable signal-to-noise by stacking the UV fluxes of galaxies out to $z \sim 0.65$. They claimed to have found no evidence for the flux evolution. I personally feel that their approach is interesting but somewhat dangerous. As I mentioned in Section 1, UV upturn galaxies are rare in the local universe where data are far more trustworthy. If I take the mean UV flux (FUV and NUV) of nearby passive (by emission line diagnostics) elliptical galaxies, it is still going to be contaminated (and even sometimes dominated) by galaxies with recent star formation, and the "mean flux" is not going to be representative as the mean flux of "true" passive galaxies devoid of young stars. This is because while RSF elliptical galaxies may be minor in number but major in flux contribution. The stochastic distribution of RSF ellipticals could contaminate the data beyond the repairable stage. Note that Yi et al. (2005) used the NUV-optical colour to find RSF contaminants. It is interesting to note that in Figure 9 of Atlee et al. $(F U V-N U V)_{o} \sim 1$ for all the data points. While they use this to suggest no UV flux evolution, this spectral slope barely qualifies the general criterion for UV upturn: $F U V-N U V<1$. I would suspect that much of the UV flux in their sample is still contaminated by recent star formation activities after their already behemoth amount of work.

\section{Epilogue}

The most significant caveat of this status report is that the database is still too small, with only 12 galaxies at $z<0.2$. Measurement errors are also too large. Ree et al. (2007) used only the brightest cluster galaxies in their analysis, but we need to look at 
other ellipticals in the same clusters as well in order to establish a general theory for the development of UV upturn. For more robust tests, we need to enlarge the sample at least by a few factors. Besides, we need to achieve an order of magnitude better signal-to-noise so that we can detect passive galaxies at $z \sim 0.3$ in the FUV where the UV flux evolution is predicted to show a turn-around. This is not going to happen using GALEX or any other instruments coming in the next few years. A more powerful instrument is desired. Until then, one might want to take the Atlee et al. approach but with a clever detective scheme for removing RSF contaminants.

With all the new findings, the UV upturn problem should be redefined.

- UV upturn is found in some giant elliptical galaxies. The fraction is probably around 10 percent when galaxies brighter than $L *$ are considered.

- Small ellipticals rarely, if at all, show a UV upturn but often show signs of recent star formation in the UV flux.

- The UV flux seems to be have been rising since the lookback time of about 2 Gyr.

- In addition, the following two old points are still valid. The Burstein et al. (1988) relation between UV flux and Mg index still seems to hold (see Martin Bureau's paper in this volume).

- The characteristic temperature of the UV sources in UV upturn galaxies is roughly 20,000-25,000K.

Theorists will be busy with more and more information from observations.

\section{References}

Atlee, D. W., Assef, R. J., \& Kochanek, C. S. 2009, ApJ, 694, 1539

Bressan, A., Chiosi, C., \& Fagotto, F. 1994, ApJS, 94, 63

Burstein, D., Bertola, F., Buson, L. M., Faber, S. M., \& Lauer, T. R. 1988, ApJ, 328, 440

Dorman, B., O'Connell, R., \& Rood, R. T. 1995, ApJ, 442, 105

Greggio, L. \& Renzini, A. 1999, Mem. S. A. It., 70, 691

Greggio, L. \& Renzini, A. 1990, ApJ, 364, 35

Han, Z., Podsiadlowski, Ph., \& Lynas-Gray, A. E. 2007, 380, 1098

Horch, E., Demarque, P., \& Pinsonneault, M. 1992, ApJ, 388, L53

Kaviraj, S. et al. 2007, ApJS, 173, 619

Lee, Y. W. 2005, ApJ, 619, L103

Martin, C. et al. 2005, 619, L1

O'Connell, R. W. 1999, ARAA, 37, 603

Peng, F. \& Nagai, D. 2009, ApJ, 705, L58

Ree, C. H. et al. 2007, ApJS, Dec. issue

Schawinski, K. et al. 2007, ApJS, 173, 512

Trager, S. C., Faber, S. M., Worthey, G., \& Gonzalez, J. J. 2000, AJ, 119, 1645

Yi, S. K. 2008, in: Ulrich Heber, C. Simon Jeffery, \& Ralf Napiwotzki (eds.), Hot Subdwarf Stars and Related Objects ASP Conference Series, Vol. 392 (ASPC: New York), p. 3

Yi, S., Demarque, P., \& Kim, Y.-C. 1997, ApJ, 482, 677

Yi, S., Demarque, P., \& Oemler, A. Jr. 1998, ApJ, 492, 480

Yi, S., Lee, Y.-W., Woo, J.-H., Park, J.-H., Demarque, P., \& Oemler, Jr. A. 1999, ApJ, 513, 128

Yi, S. et al. 2005, ApJ, 619, L111 